\title{
Effect of dimethyl sulfoxide on dentin collagen
}

\section{Mehtala, P.}

2017-08

Mehtala , P , Pashley , D H \& Tjaderhane , L 2017 , ' Effect of dimethyl sulfoxide on dentin

collagen ' , Dental Materials, vol. 33 , no. 8 , pp. 915-922 . https://doi.org/10.1016/j.dental.2017.04.018

http://hdl.handle.net/10138/297827

https://doi.org/10.1016/j.dental.2017.04.018

publishedVersion

Downloaded from Helda, University of Helsinki institutional repository.

This is an electronic reprint of the original article.

This reprint may differ from the original in pagination and typographic detail.

Please cite the original version. 


\title{
Effect of dimethyl sulfoxide on dentin collagen
}

\author{
P. Mehtälä ${ }^{a}$, D.H. Pashley ${ }^{b}$, L. Tjäderhane ${ }^{a, c, *}$ \\ ${ }^{a}$ Research Unit of Oral Health Sciences, and Medical Research Center Oulu (MRC Oulu), Oulu University Hospital \\ and University of Oulu, Oulu, Finland \\ $\mathrm{b}$ The Dental College of Georgia, Augusta University, Augusta, GA, USA \\ c Oral and Maxillofacial Diseases, University of Helsinki and Helsinki University Hospital, Helsinki, Finland
}

\section{A R T I C L E I N F O}

\section{Article history:}

Received 29 October 2016

Received in revised form

16 February 2017

Accepted 25 April 2017

\section{Keywords:}

Wettability

DMSO

Adhesion

Contact angle

Collagen expansion

Collagen shrinkage

\begin{abstract}
A B S T R A C T
Objectives. Infiltration of adhesive on dentin matrix depends on interaction of surface and adhesive. Interaction depends on dentin wettability, which can be enhanced either by increasing dentin surface energy or lowering the surface energy of adhesive. The objective was to examine the effect of dimethyl sulfoxide (DMSO) on demineralized dentin wettability and dentin organic matrix expansion.

Methods. Acid-etched human dentin was used for sessile drop contact angle measurement to test surface wetting on 1-5\% DMSO-treated demineralized dentin surface, and linear variable differential transformer (LVDT) to measure expansion/shrinkage of dentinal matrix. DMSO-water binary liquids were examined for surface tension changes through concentrations from 0 to $100 \%$ DMSO. Kruskal-Wallis and Mann-Whitney tests were used to test the differences in dentin wettability, expansion and shrinkage, and Spearman test to test the correlation between DMSO concentration and water surface tension. The level of significance was $\mathrm{p}<0.05$.

Results. Pretreatment with 1-5\% DMSO caused statistically significant concentrationdependent increase in wetting: the immediate contact angles decreased by $11.8 \%$ and $46.6 \%$ and 60 s contact angles by $9.5 \%$ and $47.4 \%$ with $1 \%$ and $5 \%$ DMSO, respectively. DMSOwater mixtures concentration-dependently expanded demineralized dentin samples less than pure water, except with high $(\geq 80 \%)$ DMSO concentrations which expanded demineralized dentin more than water. Drying times of LVDT samples increased significantly with the use of DMSO.

Significance. Increased dentin wettability may explain the previously demonstrated increase in adhesive penetration with DMSO-treated dentin, and together with the expansion of collagen matrix after drying may also explain previously observed increase in dentin adhesive bonding.
\end{abstract}

@ 2017 The Academy of Dental Materials. Published by Elsevier Ltd. All rights reserved.

\footnotetext{
* Corresponding author at: Department of Oral and Maxillofacial Diseases, University of Helsinki, P.O. Box 41, 00014 University Of Helsinki, Finland.

E-mail address: leo.tjaderhane@oulu.fi (L. Tjäderhane). http://dx.doi.org/10.1016/j.dental.2017.04.018

0109-5641/@ 2017 The Academy of Dental Materials. Published by Elsevier Ltd. All rights reserved.
} 


\section{Introduction}

Ideally, in dentin the adhesive associates intimately with surface and creates a layer which can withstand the mechanical and chemical stress imposed upon it in the oral environment. The hybrid layer where collagen and adhesive intermingle is also considered the weak link, prone to degradation over time by host-derived proteinases and hydrolysis [1,2]. Failure of fillings to perform is often due to failure in this interface [3].

The wetting ability of the adhesive is essential to the formation of a quality bond [4]. The difference in the free energies of the adhesive and solid surface dictates the manner of interaction. High energy surface is easily wetted, while low energy surface causes the adhesive to bead up on the surface. For optimal wetting solid surface energy should be maximized by surface treatments, which are usually able to expose charged groups or other high energy structures on the surface being treated [5].

In both self-etch and etch-and-rinse techniques, dentin surface is modified to enhance adhesive performance. The acid-treated dentin surface includes both crystalline apatite and collagen type I. It is a heterogeneous surface with the ability to absorb liquid and change in dimension when treated with different solvents [6]. The etched dentin presents a unique challenge to adhesive to interact with crystalline structures and organic components. Expansion of the collagen network is essential for allowing resin infiltration within and among collagen fibrils and for the formation of a good quality hybrid layer [7].

Manufacturers have tried to compose their adhesives in such a manner that their wetting ability on dentin surface is maximized. This has led to adhesives that are very hydrophilic [8]. Etched dentin modification with 2Hydroxyethyl Methacrylate (HEMA) increases wettability [9]. However, increase of hydrophilic monomer concentration leads to a hybrid layer with high water sorption [10] which is prone to hydrolytic degradation [1,3].

Dimethyl sulfoxide (DMSO) possesses a high dielectric constant combined with low surface energy and a capacity to solvate polymers [11] and adhesives [12]. DMSO is an aprotic solvent which possesses the polarity needed to break down water's self-associative tendencies and to form stable complexes with water, especially at 25-35 vol\% concentration, resulting with e.g. the lowest freezing point and viscosity $[13,14]$. DMSO is an ideal solvent to facilitate radical polymerization reactions [15] such as are used in dental adhesion. Pretreatment of acid-etched dentin with DMSO may improve long-term bond preservation $[16,17]$ by inhibition of collagenolytic enzymes [16]. It may even increase the immediate bond strength with etch-and-rinse adhesive which may be due to improved penetration of adhesive into the exposed collagen matrix [18] related to the DMSO-induced changes in dentin surface energy. To date, DMSO pretreatment has been shown to have positive effects on dentin bonds strength and its durability with very low (0.004\%) [16] and relatively high (50\%) [17] concentrations. Using all possible DMSO concentrations with conventional dentin bond testing methods, such as microtensile bond strength, would be extremely laborious and time-consuming. Therefore, we aimed to look at the effect of different DMSO concentrations on demineralized dentin behavior in search for the potential explanations that hopefully would guide the further adhesive bonding studies. The null hypotheses tested were that (1) DMSO has no effect on demineralized dentin surface energy, and that (2) DMSO has no effect on the expansion of demineralized dentin.

\section{Materials and methods}

\subsection{Specimen preparation for contact angle measurements}

Six intact human third molars extracted as part of patients' routine dental treatment were used in this study. The use of human tissue was approved by Ethical Committee of the Northern Ostrobothnia Hospital District. The teeth were stored in $0.02 \% \mathrm{NaN}_{3}$ at $4{ }^{\circ} \mathrm{C}$ until preparation. The teeth were cut with a low-speed saw (Buehler Ltd, Lake Bluff, IL, USA), exposing the superficial dentin and checked to ensure the absence of enamel.

The samples were prepared using the following series of procedures: (1) dentin surface was sanded with 500 grit abrasive paper using circular motions to achieve comparable surface roughness and smear layer; (2) dentin was etched with phosphoric acid etchant (3M ESPE, St. Paul, MN, USA) for $15 \mathrm{~s}$, with gentle agitation to achieve a uniform etched dentin surface; (3) rinsed with deionized water for $15 \mathrm{~s}$; (4) samples were set into individually prefabricated polysiloxane molds (Coltène/Whaledent AG, Altstätten, Switzerland) for stability during contact angle measurements; (5) dentin surface was treated with one of the DMSO (Sigma-Aldrich, St. Louis, MO, USA) deionized water solutions, $(0 \%, 1 \%, 2 \%, 3 \%, 4 \%$ or 5 -vol $\%$ DMSO) for $60 \mathrm{~s}$; (6) surface was dried with filtered, pressurized air for $60 \mathrm{~s}$ from approximately $1 \mathrm{~cm}$ distance; (7) after drying, contact angle measurements were conducted using deionized water.

\subsection{Contact angle measurements}

KSV Ltd. CAM 200 Surface energy analyzer (KSV Instruments Ltd, Helsinki, Finland) was used for sessile drop contact angle measurements. A manual liquid dispenser Hamilton Threaded Plunger Syringe (Hamilton Bonaduz AG, Bonaduz, Switzerland) was used for liquid disposal on dentin surface. The Young \& Laplace equation which determines the contact angle as a result of energies contributing into static system was used to determine sessile drop contact angles:

$\sigma \mathrm{SV}-\sigma \mathrm{SL}=\sigma \mathrm{LV} \cos \theta$

where $\sigma$ SV is energy in solid-vapor interface, $\sigma$ SL is energy in solid-liquid interface, $\sigma \mathrm{LV}$ is energy in liquid vapor surface and $\cos \theta$ is equilibrium contact angle. The measurement time of $61 \mathrm{~s}$ was used to allow equilibrium state to develop, but minimize the effect of evaporation on contact angles. Images were captured at $1 \mathrm{~s}$ frequency for $61 \mathrm{~s}$ using an automated trigger. For each dentin sample 10 consecutive measurements with the same pretreatment solution were performed. In between measurements, the dentin surface was rinsed with de-ionized water for $15 \mathrm{~s}$ and lightly air dried before being exposed to 


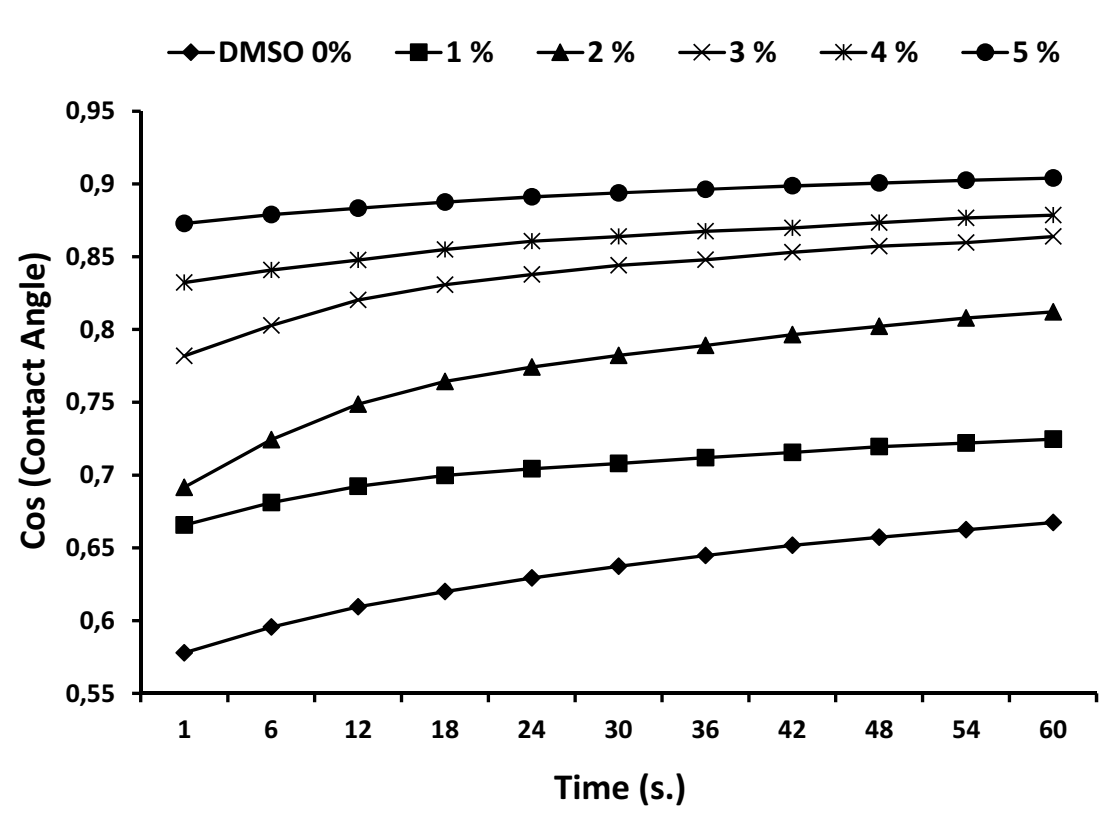

Fig. 1 - The effect of different concentrations of DMSO on acid-etched dentin over 60 s period. Wettability is presented as cosine of contact angle (mean of 10 measurements/group), where 1 equals complete wetting and 0 equals very poor wetting tendency.

a DMSO/water solution and dried for $60 \mathrm{~s}$. KSV Ltd. Software (KSV Instruments) for contact angle and surface tension measurements was used for data processing.

\subsection{DMSO/water surface tension measurements}

Several different concentrations were prepared for surface tension evaluation. Since low DMSO concentrations had significant effect on wettability, from 0 to 10 vol\% DMSO was tested with $1 \%$ intervals; from 10 to 90 vol\% testing was performed with $10 \%$ intervals. The binary liquid solutions of $\mathrm{H}_{2} \mathrm{O}$ /DMSO were evaluated using KSV CAM 200 Surface energy analyzer. The evaluated liquid was suspended at the end of the syringe tip and the drop shape is used to calculate liquid surface tension using the formula below:

$\Upsilon=\Delta \operatorname{rgR}^{2} / \beta$

where $\Upsilon$ is liquid-air surface tension $\left(\mathrm{J} / \mathrm{m}^{2}\right), \beta$ is the shape factor, $\Delta \rho$ is the difference in density between fluids at the interface $\left(\mathrm{kg} / \mathrm{m}^{3}\right), \mathrm{g}$ is gravitational acceleration $\left(\mathrm{m} / \mathrm{s}^{2}\right), \mathrm{R}$ is the radius of the drop curvature at the drop's apex $(\mathrm{m})$.

\subsection{Collagen matrix expansion measurements}

Four human teeth were used following the sample preparation and protocol previously described [19]. Briefly, mid-coronal dentin disks with an average sample thickness of $0.29 \mathrm{~mm}$ $( \pm 0.03)$ were demineralized in $0.5 \mathrm{M}$ EDTA ( $\mathrm{pH}$ 7.4) for 5 days (with stirring) at $25^{\circ} \mathrm{C}$. Fully demineralized mid-coronal dentin discs were fixed to the bottom of a polycarbonate chamber with cyanoacrylate glue. The samples within the chambers were fixed onto a linear variable differential transformer (LVDT) (Model TMS-2, EG \& G, Inc., Wellesley, MA, USA), which registers vertical dimensional changes. Dry pressurized $\mathrm{N}_{2}$ gas was used for dehydration of the samples prior application of test solutions.

For each sample a baseline height was registered using water to fully expand the demineralized dentin sample. After the initial measurement, water was aspirated and sample dried with pressurized $\mathrm{N}_{2}$ gas. When the fully dried condition was achieved, the test solvent was gently poured into the chamber and specimen expansion was allowed to plateau before registering the final height. After full expansion the solvent was aspirated and sample was rinsed with ample amount of water to wash away any remaining solvents. To measure the effect DMSO solution would have on fully expanded sample, the chamber was aspirated, but the sample was not dried with $\mathrm{N}_{2}$. Before any dimensional changes took place due to drying the solvent solution was applied to register the amount of shrinkage. Each sample was tested with each solution five times in the increasing order of DMSO concentration.

\subsection{Statistical analysis}

Statistical analysis were performed with IBM SPSS Statistics for Windows (Version 21.0. Armonk, NY, USA) for the contact angle, expansion/shrinkage and surface tension measurements. Since the data did not meet the assumption of normal distribution in all groups (Kolmogorov-Smirnov and Shapiro-Wilk tests), non-parametric methods were used. Kruskal-Wallis test with following pairwise comparison with Mann-Whitney test were used to test the differences in contact angles between different groups in the first and last recordings (1 and 61s), and the differences in water surface tension between different DMSO concentrations. Relatedsamples Wilcoxon Signed Rank test was used to analyze the differences between 1 and $61 \mathrm{~s}$ contact angles within the groups. The correlation between the DMSO concentration and 
Table 1 - Contact angles at one and $60 \mathrm{~s}$ for each solvent group (the first and last recordings: median and range).

\begin{tabular}{|c|c|c|c|c|c|}
\hline \multirow[t]{2}{*}{ DMSO } & \multicolumn{2}{|c|}{$1 \mathrm{~s}$} & \multicolumn{2}{|c|}{$60 \mathrm{~s}$} & \multirow{2}{*}{$\begin{array}{l}\text { Change } \\
\text { rate }(\% / \mathrm{s}\end{array}$} \\
\hline & Median & Range & Median & Range & \\
\hline $0 \%$ & $52.76^{\mathrm{Aa}}$ & 19.69 & $48.17^{\mathrm{Ba}}$ & 4.03 & -0.08 \\
\hline $1 \%$ & $46.05^{\mathrm{Ab}}$ & 15.65 & $43.06^{\mathrm{Bb}}$ & 6.17 & -0.05 \\
\hline $2 \%$ & $43.53^{\mathrm{Ac}}$ & 20.88 & $36.05^{\mathrm{Bc}}$ & 6.00 & -0.12 \\
\hline $3 \%$ & $38.98^{\mathrm{Ac}}$ & 15.97 & $29.94^{\mathrm{Bd}}$ & 3.35 & -0.15 \\
\hline $4 \%$ & $33.61^{\mathrm{Ad}}$ & 9.65 & $28.12^{\mathrm{Be}}$ & 5.35 & -0.09 \\
\hline $5 \%$ & $28.28^{\mathrm{Ae}}$ & 11.86 & $25.05^{\mathrm{Bf}}$ & 6.82 & -0.05 \\
\hline
\end{tabular}

Different lower case letters indicate statistically significant differences between the different DMSO concentrations (columns: Kruskal-Wallis and Mann-Whitney tests, $p<0.05$ ). Different upper case letters indicate statistically significant differences between the time points within the groups (rows; related-samples Wilcoxon Signed Rank test, $\mathrm{p}<0.01) . \mathrm{n}=10$ measurements/group.

water surface tension was tested with Spearman correlation coefficient test for the whole data (from 0 to 90 vol-\%) and separately for the 0-10 vol-\% and 0-90 vol-\% with 10\% intervals.

\section{Results}

\subsection{Contact angle and surface tension measurements}

Contact angle decreased with increasing vol-\% DMSO used to treat demineralized dentin surface. The differences in $1 \mathrm{~s}$ contact angles between the control group and all DMSOcontaining groups except $1 \%$ and $2 \%$; and between all the groups at $60 \mathrm{~s}$ were significant $(p<0.05)$ (Table 1$)$. The contact angle was significantly lower after $60 \mathrm{~s}$ than after $1 \mathrm{~s}$ in all groups $(p<0.01)$ (Table 1$)$. Fig. 1 shows the change in wetting tendency of dentin after different concentrations of DMSO as a pretreatment solvent.

Addition of DMSO into water caused changes at the liquid surface. There was a significant negative correlation between the increasing DMSO concentration and surface tension of $\mathrm{DMSO} / \mathrm{H}_{2} \mathrm{O}$ mixtures (Spearman correlation coefficient -0.954 for the 0-10 vol-\% data, -0.994 for the 0-90 vol-\% data, and -0.991 for the whole data; $p<0.001$ in all cases). Development of liquid surface tension in relation to DMSO concentration is illustrated in Fig. 2.

\subsection{Collagen matrix expansion measurement}

Water caused a rapid expansion of demineralized dentin with sharp plateau, demonstrating normal behavior of demineralized dentin in similar conditions [19]. After drying, addition of DMSO/water mixture caused the demineralized dentin sample to expand rapidly and then gradually start to plateau (Fig. 3). The expansion of collagenous matrix was slightly hindered by addition of DMSO up to 80 -vol\%, the highest reduction being seen with $40 \%-70 \%$ concentrations. $90 \%$ concentration caused a slow expansion beyond the baseline height (Table 2). Demineralized dentin samples were detached from the measuring well by the highest ( $\geq 90 \%$ ) concentrations of DMSO, thus preventing the measurement. The evaporation time required for the maximal shrinkage increased with
Table 2 - The expansion value refers to a percentage of expansion of dried demineralized sample compared to baseline height achieved by pure water $(100 \%)$.

Shrinkage value is determined by addition of specific concentration of DMSO onto wet sample and comparing the shrinkage of collagen matrix compared to baseline height.

DMSO \% Expansion/shrinkage of demineralized dentin

\begin{tabular}{lll}
\cline { 2 - 3 } & $\begin{array}{l}\text { \% Expansion } \\
\text { (median and range) }\end{array}$ & $\begin{array}{l}\text { \% Shrinkage (median } \\
\text { and range) }\end{array}$ \\
\hline $5 \%$ & $98.4(5)^{\mathrm{a}}$ & $-0.2(1)^{\mathrm{a}}$ \\
$15 \%$ & $90.7(24)^{\mathrm{ab}}$ & $-1.7(2)^{\mathrm{b}}$ \\
$20 \%$ & $83.6(7)^{\mathrm{b}}$ & $-2.9(1)^{\mathrm{b}}$ \\
$30 \%$ & $77.0(9)^{\mathrm{b}}$ & $-4.0(4)^{\mathrm{c}}$ \\
$40 \%$ & $70.7(8)^{\mathrm{c}}$ & $-6.8(4)^{\mathrm{c}}$ \\
$50 \%$ & $67.1(8)^{\mathrm{c}}$ & $-9.8(4)^{\mathrm{c}}$ \\
$60 \%$ & $66.3(4)^{\mathrm{c}}$ & $-8.4(4)^{\mathrm{c}}$ \\
$70 \%$ & $69.7(5)^{\mathrm{c}}$ & $-8.1(5)^{\mathrm{c}}$ \\
$80 \%$ & $80.5(13)^{\mathrm{b}}$ & $-5.2(4)^{\mathrm{c}}$ \\
$90 \%$ & $210.4(160)^{\mathrm{d}}$ & - \\
\hline
\end{tabular}

Different lowercase letters indicate statistically significant difference between the DMSO concentrations (Kruskal-Wallis and Mann-Whitney tests; $\mathrm{p}<0.05$ ). $\mathrm{n}=5$ measurements for both expansion and shrinkage.

the increasing concentration of DMSO. Shrinkage of the fully expanded wet collagen matrix after application of DMSO increased up to $50 \%$ and then decreased again (Table 2). DMSO/water wetted specimen did not completely shrink even after extensive drying (Fig. 3).

\section{Discussion}

We found that DMSO treatment of etched dentin significantly enhances the wettability even with the lowest concentration tested, decreasing the contact angle up to $48 \%$ after $60 \mathrm{~s}$ with $5 \%$ DMSO. The water contact angle of all DMSO treated specimens was lower than control group throughout the measured time, and the immediate contact angle changed less in all DMSO groups than in the control group (Fig. 1). These findings allow the rejection of the first hypothesis. DMSO lowers water surface tension and cohesive forces by breaking down water self-associative tendencies and thus improves the wetting tendency of the binary liquid. DMSO is a good wetting agent especially for porous irregular surfaces due to its low surface tension, high dielectric constant and perfect equilibrium between polar and surface tension properties [20]. Significantly better wetting compared to water was observed already with $1 \%$ DMSO (Table 1 ) even after drying of the dentin surface, in which the collapsed collagen network is more resistant to wetting than partially expanded hydrated dentin surface [9]. With the use of DMSO the wettability of dentin remained high.

Since DMSO in water affected the expansion of dentin organic matrix the second hypothesis is rejected. DMSO/water mixtures were able to expand dehydrated collagen matrix (Fig. 2; Table 2). Even though the maximal expansion of dentin organic matrix was lowered by DMSO up to 50-60\% DMSO and above that started to increase again, the maximal shrinkage was reduced up to $70 \%$ (Table 2) and the time required for 

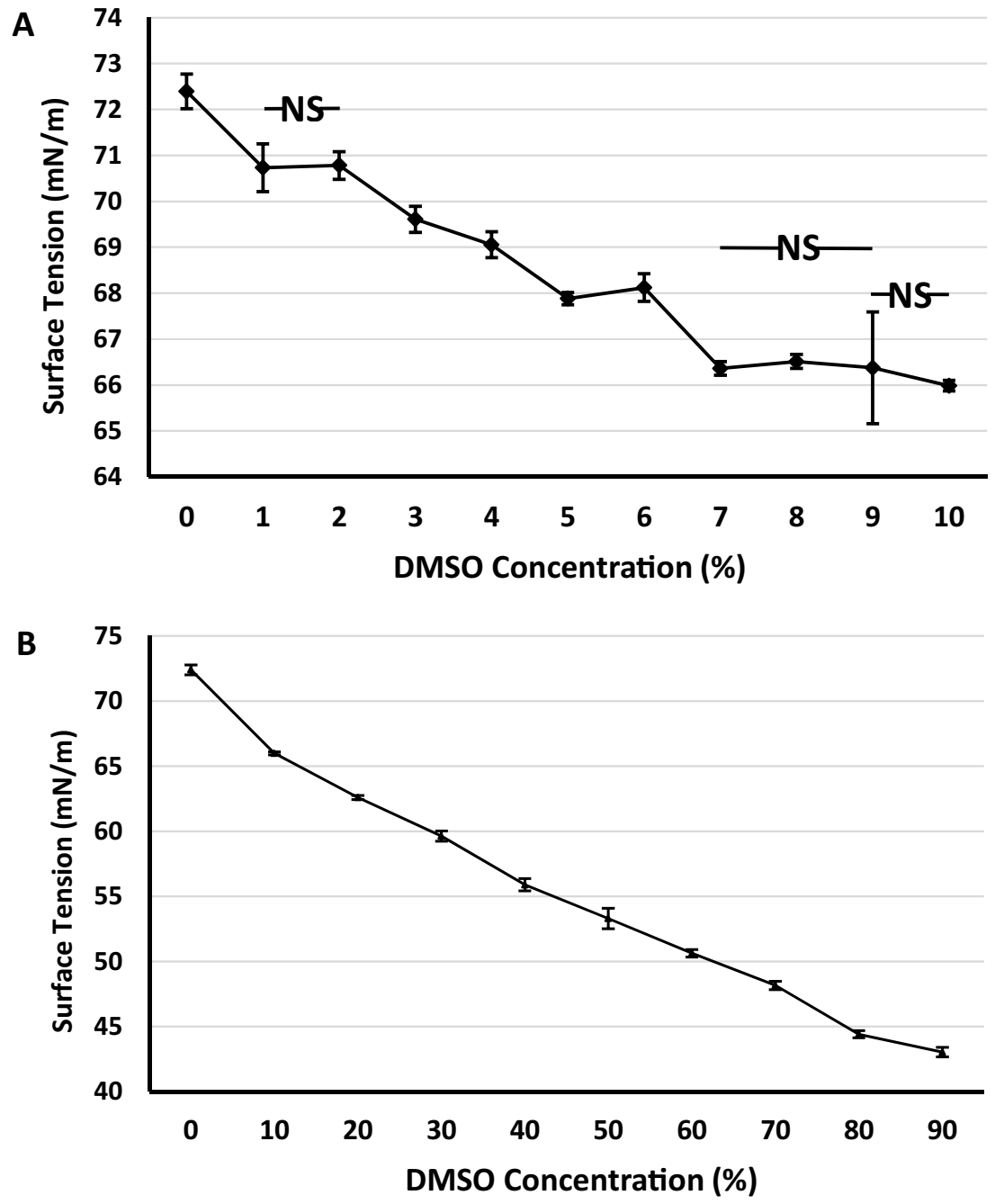

Fig. 2 - Development of binary liquid system surface tension ( $\mathrm{mN} / \mathrm{m}$ : median \pm range of 10 measurements/group) in relation to DMSO concentrations from $0 \%$ to $10 \%$ (A) and from $10 \%$ to $90 \%$ DMSO (B). The pure water surface tension is $73 \mathrm{mN} / \mathrm{m}^{3}$ and pure DMSO is $42.09 \mathrm{mN} / \mathrm{m}$ [40]. The differences between the groups were statistically significant (Kruskal-Wallis and Mann-Whitney test; $\mathrm{p}$ <.05) except between the groups indicated with vertical line and "NS" (not significant).

maximal shrinkage was markedly increased by the presence of DMSO (Fig. 3). The contact angle results may be explained in part by partially expanded collagen network by the remaining DMSO. DMSO can hydrogen-bond to proteins [21] and prevent collapse of collagen fibrils during air drying, which obliterates the interfibrillar spaces that serve as diffusion channels [19]. Faster wetting (Fig. 1) and reduced shrinkage during drying (Table 2; Fig. 3) could help to ensure that the adhesive completely covers and wets the entire prepared surface and may explain the previously observed 62-85\% deeper adhesive penetration into the exposed collagen with 2 -step self-etch and 3-step etch-and-rinse adhesive, respectively [18].

Dentin matrix expansion experiment showed that addition of DMSO onto aspirated sample caused shrinkage by up to 10 percent with $50 \%$ DMSO (Table 2). Water as a dipolar molecule with ability to self-associate (Fig. 4A, B), has a tendency to form highly structured complexes, around and within collagen fibrils $[22,23]$ and near hydrophilic structures [24]. This intimately associated and structured water has properties different than bulk water, not associated with the protein [22]. The bulk water expands the collagen matrix to its fullest state, as observed here with LVDT where drying of water-wet dentin matrix causes rapid shrinkage (Fig. 3). The removal of structured collagen-bound water (about $25 \%$ of total dentin water) cannot be removed by usual solvents and requires prolonged drying time (days) and elevated temperatures [25]. However, addition of DMSO into water breaks down water self-association and forms stable complexes where water molecules orient themselves by means of hydrogen bonds with oxygen and "hydrophobic" orientation towards methyl groups [26] (Fig. 4C), as has been recently suggested to occur with dentin collagen [17]. Thus, DMSO molecules could compete for hydrogen bond sites near and within dentin organic matrix. This could explain the shrinkage of LVDT sample when DMSO was added onto a water-wetted sample.

When DMSO/water mixture was added onto the dried collapsed LVDT sample the expansion was related to the 


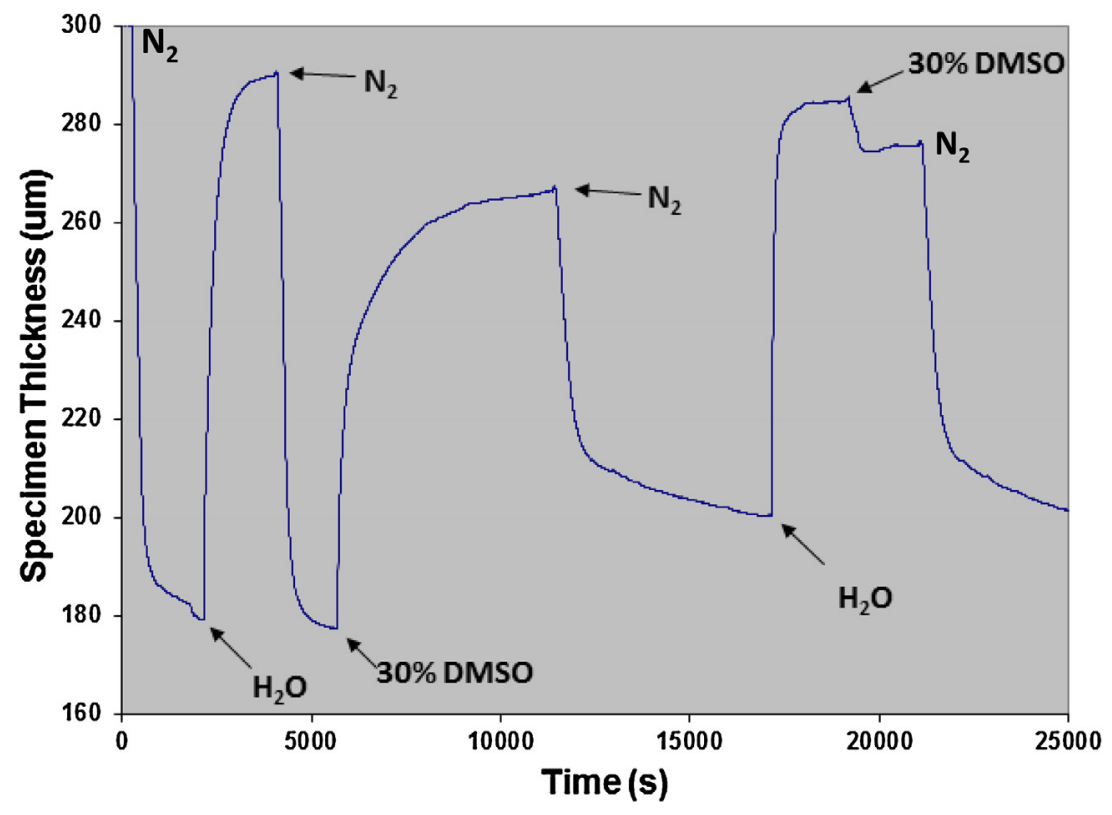

Fig. 3 - Representative data from linear variable differential transformer (LVDT) measurements, showing rapid expansion of collagenous matrix by water. Since DMSO forms stoichiometrically well-defined and stable complexes especially at $25-25 \%$ concentrations $[13,14]$, we chose $30 \%$ DMSO as a representative figure. When water hydrogen bonding was disrupted by adding 30\% DMSO in water demineralized dried sample the expansion is more gradual and did not reach the baseline expansion set by $100 \%$ water. Increased drying time after application of $30 \%$ DMSO onto the sample was observed as a more slanted downward curve at 12,500-17,000 s. Application of 30\% DMSO onto sample where was aspirated, but not dried with $\mathrm{N}_{2}$, resulted in a slight shrinkage after second application of $30 \%$ DMSO.

water concentration, but again not in a same manner as with other solvents previously tested [19]. At concentrations above $80 \%$ DMSO the expansion of collagenous matrix exceeded the expansion caused by water (Table 2). Swelling and extensibility of collagenous structure under high concentration of DMSO has previously been shown with rat tail tendon [27]. Increased spacing between the microfibrils [28], destabilization of collagen structure $[28,29]$ and disruption of intermolecular bonds [27] may be the reasons behind the matrix expansion. Water gives collagen triple helix shape and stability [30]. In high concentrations DMSO could interfere with the hydrogen bonds within the molecule by replacing water from hydrogen donor sites because DMSO-water interaction is stronger than water-water [31]. Tjäderhane et al. [16] observed the optical clearing effect of DMSO on demineralized dentin. Both optical clearing and swelling of filamentous protein require rearrangement of collagen structure.

Removal of solvents prior polymerization increases bond strength [32], but complete removal of currently used solvents is difficult or even impossible in clinically relevant conditions [33,34]. DMSO application onto dentin prior adhesion was recently shown to increase immediate and long-term bond strengths [16-18]. This is interesting due to the fact that complete evaporation of DMSO from dentin surface within clinically relevant time is not likely. On the contrary, DMSO applied on etched dentin surface would rather increase in concentration if air dried, yet the immediate and especially long-term bond strength are rather improved than decreased [16-18]. It is tempting to speculate that DMSO could serve as an adhesive solvent that does not need to be evaporated, which should reduce the technique sensitivity of the adhesive bonding.

Introduction of new solvents should take into account their suitability as solvents and ability to expand exposed collagen on dentin surface. Since DMSO is able to expand collagen matrix, solvates adhesive components [12], infiltrates dentinal collagen [35], has low toxicity [11,36,37] and has been indicated to improve both immediate and long-term bond strength [16-18] make DMSO a possible new solvent for dental adhesives to enhance wetting. Water causes phase separation, because of the polar nature it associates strongly with charged groups of other molecules and with itself causing droplet formation within the adhesive [34] and repelling hydrophobic adhesive components [38]. Introduction of DMSO into adhesive formulation could reduce the amount of water, HEMA and other solvents needed to wet and infiltrate the etched dentin surface, possibly allowing the use of less hydrophilic monomers. Adding a solvent with a capacity to solvate hydrophobic monomer components could improve adhesive performance by increasing the degree of conversion and decreasing nanoleakage caused by water [34,39]. DMSO could also help keep the components solvated and formulation more stable. Further studies are needed to evaluate the effect DMSO has on dentin collagen and if DMSO could be useful as a solvent added into the adhesives. 

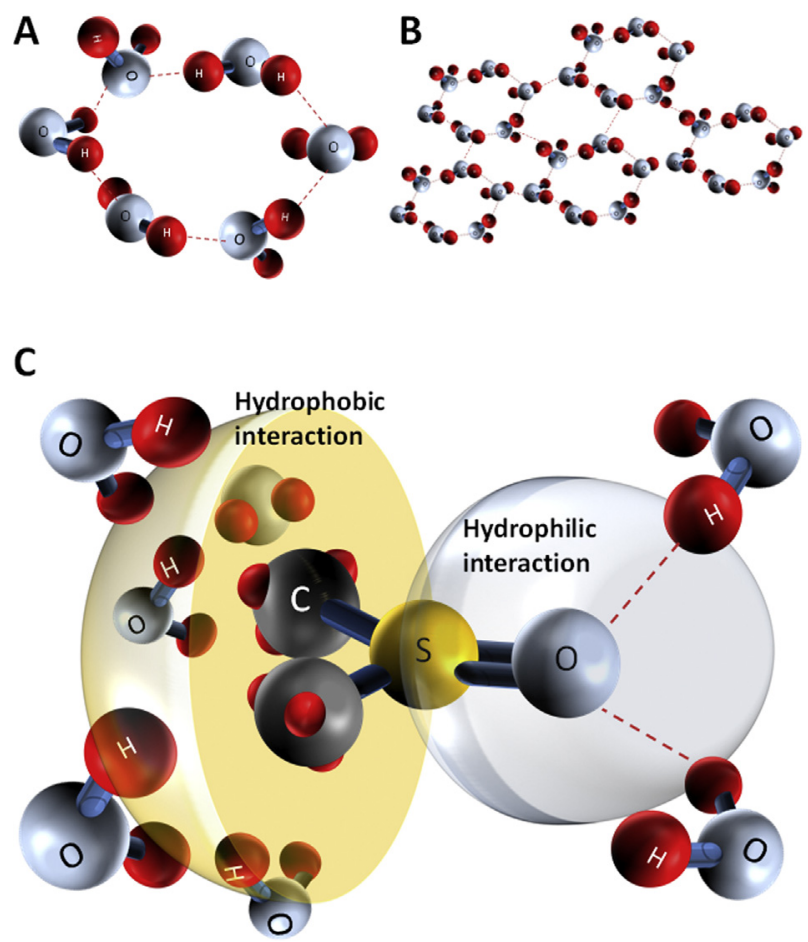

Fig. 4 - The effect of DMSO on the behavior of water molecules. Thin red lines represent hydrogen bonds between the molecules. (A,B) Naturally occurring water in highly structured form. (C) Addition of DMSO molecule causes the breakage of the water self-association because DMSO-water interaction is $\mathbf{1 . 3}$ times stronger than between water molecules. DMSO molecule with one oxygen atom is able to form a hydrogen bond with two $\mathrm{H} 2 \mathrm{O}$ molecules in a hydrophilic manner. Methyl $\left(\mathrm{CH}_{3}\right)$ groups possess no charge and water orients itself in a lattice around it in a hydrophobic manner. O: oxygen. $\mathrm{H}$ : hydrogen. S: sulfur; C: carbon.

(Modified from Bellossent-Funel 1998 [41]).

\section{Acknowledgments}

The authors thank Ms Kelli Agee for her expert help in LVDT experiments. The study was financially supported by the Academy of Finland and the Finnish Dental Society Apollonia.

\section{REFERENCES}

[1] Tjäderhane L, Nascimento FD, Breschi L, Mazzoni A, Tersariol IL, Geraldeli S, et al. Optimizing dentin bond durability: control of collagen degradation by matrix metalloproteinases and cysteine cathepsins. Dent Mater 2013;29:116-35

[2] Tjäderhane L, Nascimento FD, Breschi L, Mazzoni A, Tersariol IL, Geraldeli S, et al. Strategies to prevent hydrolytic degradation of the hybrid layer-a review. Dent Mater 2013;29:999-1110.

[3] Pashley DH, Tay FR, Breschi L, Tjäderhane L, Carvalho RM, Carrilho M, et al. State of the art etch-and-rinse adhesives. Dent Mater 2011;27:1-16.
[4] Marshall SJ, Bayne SC, Baier R, Tomsia AP, Marshall GW. A review of adhesion science. Dent Mater 2010;26:11-6.

[5] Awaja F, Gilbert M, Kelly G, Fox B, Pigram PJ. Adhesion of polymers. Prog Polym Sci 2009;34:948-68.

[6] Eddleston CL, Hindle AR, Agee KA, Carvalho RM, Tay FR, Rueggeberg FA, et al. Dimensional changes in acid-demineralized dentin matrices following the use of HEMA-water versus HEMA-alcohol primers. J Biomed Mater Res A 2003;67:900-7.

[7] Pashley DH, Tay FR, Carvalho RM, Rueggeberg FA, Agee KA, Carrilho M, et al. From dry bonding to water-wet bonding to ethanol-wet bonding. A review of the interactions between dentin matrix and solvated resins using a macromodel of the hybrid layer. Am J Dent 2007;20:7-20.

[8] Tay FR, Pashley D. Have dentin adhesives become too hydrophilic? J Can Dent Assoc 2003;69:726-31.

[9] Hitmi L, Bouter D, Degrange M. Influence of drying and HEMA treatment on dentin wettability. Dent Mater 2002;18:503-11.

[10] Malacarne J, Carvalho RM, de Goes MF, Svizero N, Pashley DH, Tay FR, et al. Water sorption/solubility of dental adhesive resins. Dent Mater 2006;22:973-80.

[11] Roy K-M. Sulfones and sulfoxides. In: Ullmann's encyclopedia of industrial chemistry; 2000, http://dx.doi.org/10.1002/14356007.a25_487/abstract [Accessed 8 November 2013].

[12] Grégoire G, Dabsie F, Dieng-Sarr F, Akon B, Sharrock P. Solvent composition of one-step self-etch adhesives and dentine wettability. J Dent 2011;39:30-9.

[13] Vishnyakov A, Lyubartsev AP, Laaksonen A. Molecular dynamics simulations of dimethyl sulfoxide and dimethyl sulfoxide-water mixture. J Phys Chem A 2001;105: 1702-10.

[14] Catalán J, Diaz C, Garcia-Blanco F. Characterization of binary solvent mixtures of DMSO with water and other cosolvents. J Org Chem 2001;66:5846-52.

[15] Martin D, Weise A, Niclas H. The solvent dimethyl sulfoxide. Angew Chem Int Ed 1967;6:318-34.

[16] Tjäderhane L, Mehtälä P, Scaffa P, Vidal C, Pääkkönen V, Breschi L, et al. The effect of dimethyl sulfoxide (DMSO) on dentin bonding and nanoleakage of etch-and-rinse adhesives. Dent Mater 2013;29:1055-62.

[17] Stape TH, Tjäderhane L, Tezvergil-Mutluay A, Yanikian CR, Szesz A, Loguercio AD, et al. Dentin bond optimization using the dimethyl sulfoxide-wet bonding strategy: a 2-year in vitro study. Dent Mater 2016;32:1472-81.

[18] Stape TH, Tjäderhane L, Marques MR, Aguiar FH, Martins LR. Effect of dimethyl sulfoxide wet-bonding technique on hybrid layer quality and dentin bond strength. Dent Mater 2015;31:676-83.

[19] Pashley DH, Agee KA, Nakajima M, Tay FR, Carvalho RM, Terada RS, et al. Solvent-induced dimensional changes in EDTA-demineralized dentin matrix. J Biomed Mater Res 2001;56:273-81.

[20] Redón R, Vázquez-Olmos A, Mata-Zamora M, Ordóñez-Medrano A, Rivera-Torres F, Saniger J. Contact angle studies on anodic porous alumina. J Coll Interface Sci 2005;287:664-70

[21] Zheng Y-A, Ornstein RL. A molecular dynamics and quantum mechanics analysis of the effect of DMSO on enzyme structure and dynamics: subtilisin. J Am Chem Soc 1996;118:4175-80.

[22] Hoeve CAJ, Tata AS. The structure of water absorbed in collagen. J Phys Chem 1978;82:1660-3.

[23] Bella J, Brodsky B, Berman HM. Hydration structure of a collagen peptide. Structure 1995;3:893-906.

[24] Chai B, Pollack GH. Solute-free interfacial zones in polar liquids. J Phys Chem B 2010;114:7626-52. 
[25] Agee KA, Prakki A, Abu-Haimed T, Naguib GH, Nawareg MA, Tezvergil-Mutluay A, et al. Water distribution in dentin matrices: bound vs unbound water. Dent Mater 2015;31:205-16.

[26] Mancera RL, Chalaris M, Samios J. The concentration effect on the 'hydrophobic' and 'hydrophilic' behaviour around DMSO in dilute aqueous DMSO solutions. A computer simulation study. J Mol Liq 2004;110:147-53.

[27] Gries G, Bublitz G, Lindner J. The effect of dimethyl sulfoxide on the components of connective tissue (clinical and experimental investigations). Ann N Y Acad Sci 1967;141:630-7.

[28] Zimmerley M, McClure RA, Choi B, Potma EO. Following dimethyl sulfoxide skin optical clearing dynamics with quantitative nonlinear multimodal microscopy. Appl Opt 2009;48:D79-87.

[29] Bui AK, McClure RA, Chang J, Stoianovici C, Hirshburg J, Yeh AT, et al. Revisiting optical clearing with dimethyl sulfoxide (DMSO). Lasers Surg Med 2009;41:142-8.

[30] Bertassoni LE, Orgel JP, Antipova O, Swain MV. The dentin organic matrix-limitations of restorative dentistry hidden on the nanometer scale. Acta Biomater 2012;8:2419-33.

[31] Kirchner B, Reiher M. The secret of dimethyl sulfoxide-water mixtures: a quantum chemical study of $1 \mathrm{DMSO}-\mathrm{nWater}$ clusters. J Am Chem Soc 2002;124:6206-15.

[32] Ikeda T, De Munck J, Shirai K, Hikita K, Inoue S, Sano H, et al Effect of air-drying and solvent evaporation on the strength of HEMA-rich versus HEMA-free one-step adhesives. Dent Mater 2008;24:1316-23.
[33] Nihi FM, Fabre HS, Garcia G, Fernandes KB, Ferreira FB, Wang L. In vitro assessment of solvent evaporation from commercial adhesive systems compared to experimental systems. Braz Dent J 2009;20:396-402.

[34] Yiu CK, Pashley EL, Hiraishi N, King NM, Goracci C, Ferrari M, et al. Solvent and water retention in dental adhesive blends after evaporation. Biomaterials 2005;26:6863-72.

[35] Takahashi M, Nakajima M, Tagami J, Scheffel DL, Carvalho RM, Mazzoni A, et al. The importance of size-exclusion characteristics of type I collagen in bonding to dentin matrices. Acta Biomater 2003;9:9522-8.

[36] Marren K. Dimethyl sulfoxide: an effective penetration enhancer for topical administration of NSAIDs. Phys Sportsmed 2011;39:75-82

[37] Hebling J, Bianchi L, Basso FG, Scheffel DL, Soares DG Carrilho MRO, et al. Cytotoxicity of dimethyl sulfoxide (DMSO) in direct contact with odontoblast-like cells. Dent Mater 2015;31:399-405.

[38] Ye Q, Wang Y, Spencer P. Nanophase separation of polymers exposed to simulated bonding conditions. J Biomed Mater Res B Appl Biomater 2009;88:339-48.

[39] Paul SJ, Leach M, Rueggeberg FA, Pashley DH. Effect of water content on the physical properties of model dentine primer and bonding resins. J Dent 1999;27:209-14.

[40] Wohlfarth C, Wohlfarth B. Surface tension of pure liquids and binary liquid mixtures. Berlin: Springer Berlin Heidelberg; 2008.

[41] Bellossent-Funel M-C. Hydration processes in biology. Washington D.C: IOS Press; 1998 